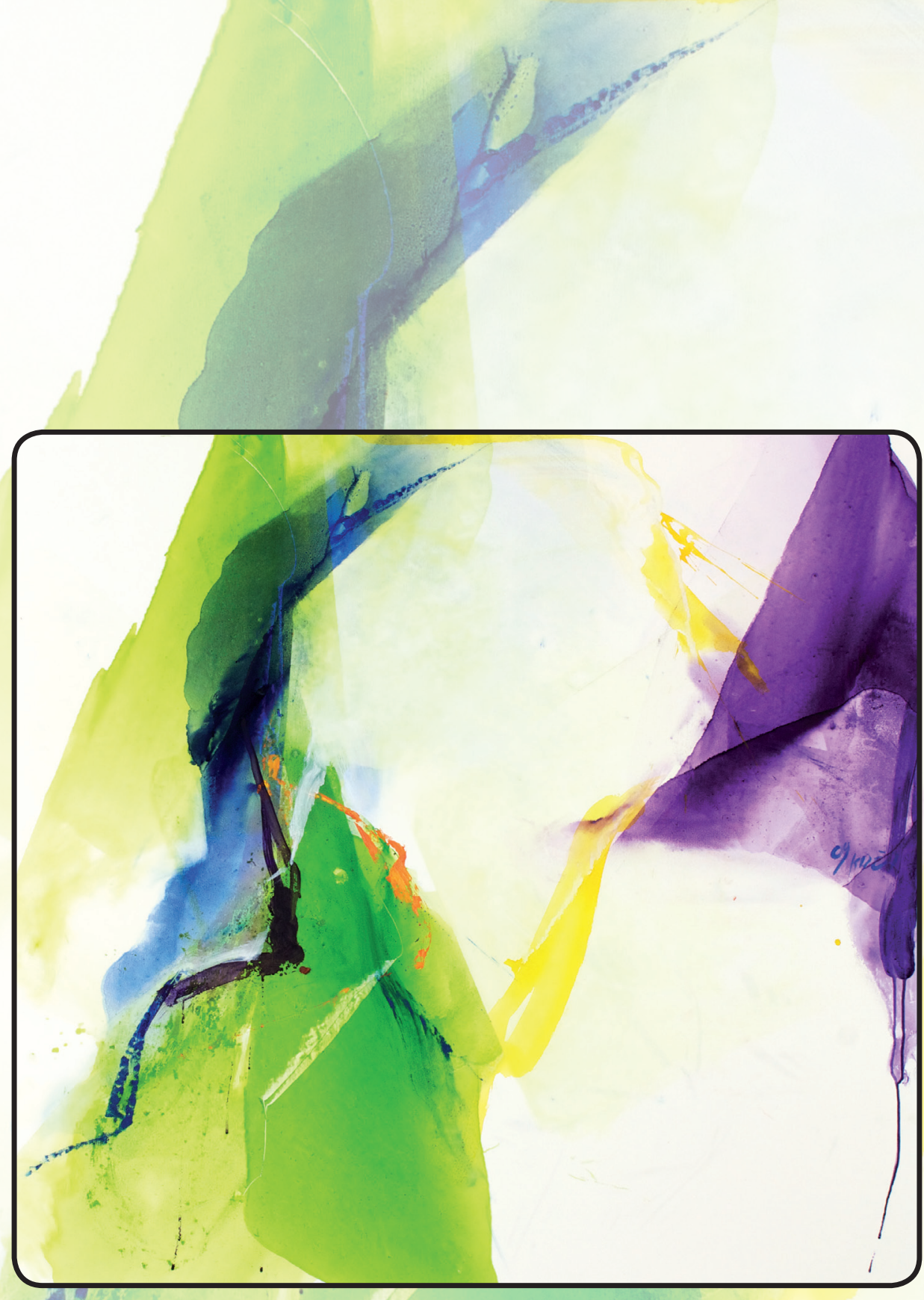




\section{Hommage Aux 12 apotres}

Óleo sobre lienzo

$120 \times 120 \mathrm{cms}$

2009

Colección Privada

Universidad Santo Tomás 


\title{
DERECHOS DERIVADOS DEL CONSENTIMIENTO INFORMADO EN LA PRESTACIÓN DEL SERVICIO MÉDICO EN COLOMBIA
}

\section{Rafael Enrique López Camargo}

Maestría en Derecho Administrativo - Universidad Libre, (Col), (en curso); Especialización Derecho Comercial - Universidad Libre (Col); Abogado-Universidad Libre (Col); Licenciado en Ciencias de la Educación Ciencias Sociales y Económicas - Universidad Pedagógica y Tecnológica de Colombia (UPTC); Docente Universidad Santo Tomás - Seccional Tunja (Col).

E-mail: enriquelopezc8@hotmail.com

\begin{abstract}
Resumen
Lo novedoso surgido en bioética médica tiene que ver con la inclusión del concepto consentimiento informado, expresado por el paciente y que está regulado en todos los países del mundo y, desde luego, en la legislación colombiana, con la adopción rigurosa de los protocolos médicos. Igualmente, se plantea el aporte jurídico a la actividad médica, como lo es la aparición consecuente y necesaria de algunos Derechos Fundamentales que deben respetarse en los pacientes; el derecho a la vida y a la salud, a la autodeterminación, a la libertad religiosa y de conciencia y a morir con dignidad, entre otros.
\end{abstract}

\section{Palabras Clave}

Responsabilidad médica, consentimiento informado, derechos, salud, medicina, protocolos, autodeterminación.

\begin{abstract}
The novel appeared in medical bioethics relates to the inclusion concept of informed consent, expressed by the patient and that is regulated in every country in the world and certainly in Colombian law, with the adoption of strict medical protocols. Equal raises the legal contribution to the medical activity, as is required and the consequent emergence of some fundamental rights to be observed in patients, to life and health, to self-determination, freedom religion and conscience and to die with dignity, among others.
\end{abstract}

\section{Key Words}

Responsibility medical, informed consent, rights, health, medicine, protocols, selfdetermination. 



\section{DERECHOS DERIVADOS DEL CONSENTIMIENTO INFORMADO EN LA PRESTACIÓN DEL SERVICIO MÉDICO EN COLOMBIA*}

Rafael Enrique López Camargo

La prestación del servicio médico, implica responsabilidad civil, contractual y extracontractual por parte del galeno y consecuencialmente el Estado debe resarcir los daños por su agente, si este se encuentra vinculado a aquél, y la competencia para conocer del asunto le corresponde al Juzgado Administrativo o Tribunal Administrativo de la Entidad Territorial, donde la falla se produjo; cuando se trate de relaciones médico-paciente, entre particulares, es decir, cuando el prestador de la salud sea una entidad privada, esos procesos se ventilarán a través de la jurisdicción ordinaria, valga decir, jueces civiles, municipales o del circuito y Tribunal Superior de Distrito Judicial, según sea el monto de las prestaciones.

Existen diversas teorías que buscan, de alguna manera, proteger a la víctima del daño; así, pues, tenemos: la responsabilidad del Estado por falla en la prestación del servicio; la falla presunta; la teoría de la garantía, en razón de considerarse la actividad médica como peligrosa; la culpa probada; la falla probada del servicio.

En el presente artículo se reflexiona sobre la inclusión del concepto Consentimiento Informado, se plantea el aporte jurídico a la actividad médica, como lo es la aparición consecuente y necesaria de algunos Derechos Fundamentales. El texto se enmarca en el Proyecto: Responsabilidad del Estado por hecho médicos; Línea de investigación en Responsabilidad del Estado y Derecho Administrativo, del Grupo de Investigaciones del Centro de Investigaciones Socio - Jurídicas de la Universidad Santo Tomás - Tunja, (Col). 
Dichas teorías llevarían, en un primer momento, a hallar culpable al médico, sin embargo, existe un eximente de responsabilidad o mejor de culpabilidad de éste y encuentra su basamento en la culpa exclusiva de la víctima y la presunción de falla que se presenta cuando los profesionales de la salud y las EPS obraron con diligencia y cuidado y se proporcionó al paciente los especiales conocimientos para preservar su salud.

La doctrina y la jurisprudencia moderna han sostenido la teoría del consentimiento informado, mediante el cual el paciente recibe de parte del médico una información precisa, clara y suficiente para que aquél decida de manera autónoma y libre someterse a un tratamiento médico, terapéutico o quirúrgico. Esta información le permitirá aceptar o rechazar los procedimientos, ya que ella adquiere especial importancia, como actividad preventiva del galeno, así como trascendencia probatoria, si tiempo después de la intervención, llegare a presentarse algún accidente.

Los médicos y doctrinantes, doctores Juan Pablo Barreneche y Julio César Galán Cortés, coinciden en manifestar que: "el consentimiento informado es el fruto del paso de beneficencia a autonomía y una de las máximas aportaciones que el derecho ha hecho a la medicina",

Desde el surgimiento de este término del consentimiento informado en 1957, se ha dado la importancia necesaria al principio de la autonomía del paciente, quien en virtud de éste delega en el médico la responsabilidad de su tratamiento, dándose así un giro de 180 grados en la neo-ética médica, que de la esfera de lo personal se transporta a lo social, en razón de que este consentimiento muchas veces es otorgado por sus familiares y parientes cercanos. Este principio y derecho a la vez que tiene el paciente a la libre autodeterminación, contempla la posibilidad de retirar el consentimiento de forma libre cuando así lo solicite, previo el diligenciamiento de los protocolos médicos necesarios.

En Colombia la legislación correspondiente, ha reglamentado tímidamente sobre ética médica en la ley 23 de 1981 (Código de Ética Médica); por manera de ejemplo, el artículo 15 de la citada ley precisa lo siguiente:

"el médico no expondrá a su paciente a riesgos injustificados. Pedirá su consentimiento para aplicar los tratamientos médicos y quirúrgicos que considere indispensables y que puedan afectarlo física y psíquicamente, salvo en los casos en que ello no fuere posible, y le explicara al paciente o a sus responsables de tales consecuencias anticipadamente."

Y, no se precisó nada sobre la autodeterminación del paciente.

Ahora bien, del consentimiento informado se deriva una serie de relaciones con derechos tales como el derecho a la vida y a la salud, el derecho a la autodeterminación, el derecho a la libertad religiosa y de conciencia y el derecho a morir con dignidad, (eutanasia) entre otros, los cuales le son inherentes al paciente, y de los que puede hacer uso ya se trate por cuestiones de fe y conciencia o bien por principios de dignidad humana, que no admite su desconocimiento. 
La responsabilidad por la prestación del servicio médico corresponde asumirla a la Entidad tal como lo prevé la Constitución Política; la carga de la prueba en esta clase de procesos, incumbe a las partes, tal como lo establece el Art. 177 del $\mathrm{CPC}$; pero, en grado especial, será el médico quien tendrá que probar, que proveyó la información necesaria, oportuna y clara a su paciente, sobre su estado de salud y las consecuencias que se generarían por su tratamiento lo que lo llevaría finalmente a confiar en su médico y expresar con plena madurez y libremente su voluntad.

\section{Derechos derivados del consentimiento informado en la prestación del servicio médico en Colombia}

\section{Aparición del consentimiento informado}

El concepto de consentimiento informado en la prestación del servicio médico, es de reciente aparición y no es otra cosa que el derecho que tiene el paciente a la libre autodeterminación, frente a una consulta médica o tratamiento terapéutico y/o intervención quirúrgica.

Este término, de aplicación y connotación eminentemente jurídicas, pasa al campo de la ética médica, con el objeto de prevenir responsabilidades civiles en las que puedan incurrir los profesionales de la salud, por la omisión de información al enfermo o familiares cercanos. Al respecto el doctor Julio César Galán Cortés ${ }^{1}$ afirma que el consentimiento informado es una de las máximas aportaciones que el derecho ha hecho a la medicina, que es un hecho incontrovertible, que el consentimiento informado es ajeno a la tradición médica, que lo ha desconocido a lo largo de su historia, si bien, en la actualidad constituye un presupuesto esencial de la relación médico-paciente, lo que redundará en una significativa mejora de la calidad asistencial.

Precisamente, las primeras condenas contra médicos por no advertir a sus pacientes los riesgos que enfrentarían por las intervenciones quirúrgicas, se dieron en los Estados Unidos de Norteamérica en 1957 en el caso Martín Salgo vs. Leland Stanford, quien sufrió parálisis permanente luego del tratamiento traslumbrar a que fue sometido por su médico. Así mismo, el Consejo de Estado Francés en enero 15 del año 2001, profirió condena contra la administración pública porque se practicó a un paciente una angioplastia, sin informarle los riesgos que este procedimiento terapéutico tenía para su salud ${ }^{2}$.

Gran desarrollo jurisprudencial ha tenido el consentimiento informado en los Estados Unidos; en el juicio Natanson vs. Kline, la Corte Suprema de Justicia

1 GALÁN CORTÉS, Julio César. Doctor en Medicina, Abogado Universidad de Oviedo. España.

2 El comentario al fallo mencionado hecho por Denis DENDONCKER puede leerse en: La apreciación de la responsabilidad en caso de falta de información al paciente. 
responsabilizó al médico que le practicó la mastectomía, por las consecuencias irreparables que sufrió en su hemitórax izquierdo derivadas de las profundas y extensas quemaduras por recibir terapias con cobalto.

En Colombia se ha reglamentado tímidamente sobre ética médica en la ley 23 de 1981 (Código de Ética Medica); así, a manera de ejemplo el artículo 15 de la citada ley precisa lo siguiente:

"el médico no expondrá a su paciente a riesgos injustificados. Pedirá su consentimiento para aplicar los tratamientos médicos y quirúrgicos que considere indispensables y que puedan afectarlo física y psíquicamente, salvo en los casos en que ello no fuere posible, y le explicara al paciente o a sus responsables de tales consecuencias anticipadamente".

Y, no se precisó nada sobre la autodeterminación del paciente.

El médico Julio Enrique Peña B, destacado doctrinante colombiano en la rama de la salud, define el consentimiento informado en los siguientes términos:

"es el procedimiento mediante el cual el médico comunica e informa a su paciente las diversas opciones y alternativas para el diagnóstico, evolución y tratamiento de la enfermedad, advirtiéndole los riesgos inherentes a los procedimientos, así como los beneficios que se deriven en forma clara, que le permita hacer una decisión racional, en últimas es la autorización autónoma de una intervención médica de cada paciente en particular".

Para resolver este vacío legal sobre la autodeterminación del paciente, se expidió por parte del Ministerio de Salud la resolución de 13437 de 1981 que contempla dentro del decálogo del paciente el derecho a

"disfrutar de una comunicación plena y clara con el médico, apropiada a sus condiciones sicológicas y culturales que le permitan obtener toda la información necesaria respecto a la enfermedad que padece, así como los procedimientos $\mathrm{y}$ tratamientos que se le vayan a practicar y al pronóstico y riesgos que dicho tratamiento conlleve. También su derecho a que él y sus familiares o representantes en caso de inconsciencia o minoría de edad, consientan o rechacen estos procedimientos, dejando expresa constancia ojalá escrita de su decisión".

Desde el surgimiento de este término del consentimiento informado en 1957, se ha dado la importancia necesaria al principio de la autonomía del paciente, quien en virtud de éste delega en el médico la responsabilidad de su tratamiento, dándose así, un giro de 180 grados en la neo-ética médica, que de la esfera de lo personal se transporta a lo social. Este principio y derecho a la vez que tiene el paciente a la libre autodeterminación, contempla la posibilidad de retirar el consentimiento de forma libre cuando así lo solicite. 


\section{Elementos que componen el consentimiento informado $(C I)^{3}$}

Voluntariedad (libertad). Tiene implicaciones subjetivas por parte del paciente quien ha pasado a ocupar una posición más activa en esta relación y que puede decidir si acepta o no determinado tratamiento, teniendo claramente presentes las consecuencias que se derivan de su decisión y que lo condujeron a retirar en un momento dado su consentimiento. El Art. 1502 del CCC señala, que para que una persona se obligue a otra, por declaración de voluntad, es necesario que consienta dicho acto o declaración de voluntad y su consentimiento debe estar exento de vicios.

Información en cantidad y con calidad suficiente. La información debe darse en el contexto adecuado, en el momento idóneo y con el tacto necesario en cada caso $^{4}$. La información y comunicación al paciente juegan papel preponderante ya que a partir de allí es donde se da una plena relación que busca aliviar las dolencias de un enfermo. La jurisprudencia francesa ${ }^{5}$, ha decidido que un médico tiene una obligación particular de información hacia su paciente y le incumbe probar que ha cumplido con esta obligación. El deber de información se ha vuelto un arma fatal: el médico nunca podrá probar, que ha dado en lenguaje suficientemente claro, para ser comprendido, todas las informaciones concernientes a todos los riesgos a los que el paciente está expuesto.

Para que el paciente decida libremente someterse a un tratamiento médico o procedimiento terapéutico, debe tener el suficiente conocimiento sobre los riesgos que ha de enfrentar, es por ello que se requiere que el médico le proporcione una información cierta, precisa y completa, que le permita aceptar o rechazar los procedimientos recomendados; luego, cuando el paciente acepta lo planteado por el galeno, se presenta un consentimiento idóneo, que lo ha llevado a confiar plenamente en su médico y expresar libremente su voluntad.

Validez y autenticidad de la decisión. El paciente debe gozar de plena capacidad de juicio y discernimiento para tomar con la mayor madurez una decisión que tiene implicaciones futuras para su salud y que, consecuentemente, libere al galeno de responsabilidad alguna. Cuando se trate de un menor de edad, o persona mayor que no tenga la capacidad a causa de una disfunción mental o consecuencia de una enfermedad, que la lleve a expresar con madurez suficiente su consentimiento, sólo podrá ser intervenida quirúrgicamente con la autorización de su representante legal o, en su defecto, por una autoridad legal prevista para el efecto.

3 REVISTA CUBANA DE ENFERMERÍA, (2006). REYES LOPEZ, Madelin et al. Consentimiento informado en pacientes sometidos a cirugía por mínimo acceso. Rev Cubana Enfermer [online]. 2006, vol.22, n.4, pp. 0-0. ISSN 0864-0319.

4 FRANCESC, A. (1995). Comités de Bioética: necesidad, estructura y funcionamiento. Labor hospitalaria. 40, 229; 136

5 Europa, Tribunal Supremo, (1997, febrero), Francia. 
Respecto del alcance de la voluntad de los terceros a favor de menores la Corte Constitucional ${ }^{6}$ ha expresado:

\begin{abstract}
"en casos determinados es legítimo que los padres y el Estado puedan tomar ciertas medidas a favor de los menores, incluso contra la voluntad aparente de estos últimos, puesto que se considera que éstos aún no han adquirido la suficiente independencia de criterio para diseñar autónomamente su plan de vida y tener propia conciencia de sus intereses. Esto es lo que justifican instituciones como la patria potestad o la educación primaria obligatoria, pues si los menores no tienen capacidad jurídica para consentir, otros deben y pueden hacerlo en su nombre y para proteger sus intereses".
\end{abstract}

\title{
Derechos derivados del Consentimiento Informado
}

Lo novedoso surgido en bioética médica tiene que ver con la gama de Derechos derivados del consentimiento informado, que no están planteados en la legislación colombiana, a pesar de la aparente rigurosidad con que se tramitan los protocolos médicos. Igualmente, se plantea el aporte jurídico a la actividad médica, como la aparición consecuente y necesaria de algunos Derechos Fundamentales que deben respetarse en los pacientes. El derecho a la vida y a la salud, a la autodeterminación, a la libertad religiosa y de conciencia y a morir con dignidad, entre otros.

El derecho a la autodeterminación: El principio de la autodeterminación lo hemos tratado en la parte inicial del presente escrito, pero se hace necesario precisar o ahondar en otros aspectos no menos importantes. El tratadista Max Charlesworth ${ }^{7}$ plantea respecto del consentimiento y autonomía:

"El derecho a la autonomía o autodeterminación es de hecho el fundamento de todos los demás derechos humanos ya que no tendría ningún sentido hablar de "derechos" a no ser que seamos capaces de decidir por nosotros mismos y ser responsables de nuestra vida"

El principio autonomista propende por el respeto a las decisiones libres y voluntarias de los pacientes, aún aceptando aquellas que en un momento dado pongan en riesgo su salud e incluso la vida, llevando a éstos a decidir con excesivo individualismo, en detrimento de la obligación natural que cobija al médico cual es la de procurar la mejoría o curación del paciente ${ }^{8}$.

Es menester precisar que la corriente autonomista surge en contraposición a la corriente paternalista o asistencialista y que la Constitución política de Colombia de 1991, recoge al contemplar la concertación del médico con el paciente sobre el tratamiento que se la va a ofrecer.

6 Colombia, Corte Constitucional, (1995) Sentencia T-477, M. P. Martínez Caballero, A. Bogotá.

7 Charlesworth, M. (1996). La bioética en una sociedad liberal. Edit. Cambridge University Press. Trad. Mercedes González.

8 Franco Zuloaga, J. A., \& Agudelo Yepes, M. C. (año). El consentimiento y la advertencia de riesgo en medicina, Revista Instituto Nacional de Medicina en Colombia Vol. XVI 
"Esta relación tiene dos componentes, uno de carácter técnico-científico y otro de carácter volitivo. El primero comprende el conocimiento, la experiencia y el buen juicio del galeno, mientras que en el segundo está expresada la voluntad del paciente, preservando de esta manera la autodeterminación y la dignidad humana".

EI derecho a la libertad religiosa y de conciencia: Muy seguramente es motivo de extrañeza, que cuando un paciente acuda a una EPS, ya se trate por consulta externa o bien por remisión al galeno especialista, el dependiente de la institución le pregunte sobre su confesión religiosa; esta situación sólo busca identificar y valorar la tendencia confesional del paciente, para efectos de practicar los tratamientos legal y médicamente permitidos pero, también, tener en cuenta las consideraciones que en materia religiosa deban hacerse.

Sabido es que algunas creencias religiosas no comulgan con tratamientos terapéuticos o quirúrgicos en los cuales sea necesario realizar la transfusión de sangre. A título de ejemplo existen los fieles testigos de Jehová, personas que obedeciendo a sus principios religiosos se niegan a consentir procedimiento tranfusional alguno, sobreponen por encima del derecho a la vida, el derecho a creer en dogmas religiosos. Situación como la aquí planteada, no sólo pone ante una disyuntiva al médico, sino ante un real apuro profesional que de no sortearse satisfactoriamente lo puede ver implicado en una investigación ético-médica o incluso enfrentar en los estrados judiciales una investigación penal.

¿Qué sucedería en el caso de un médico que desacatando preceptos de orden religioso logre salvar la vida de un paciente mediante el procedimiento de la transfusión sanguínea? Desde el punto de vista ético, esta situación podría llevar al médico a ser investigado por el Consejo de Ética Médica y la Procuraduría General de la Nación, para determinar responsabilidades en este sentido, aunque resulte verdaderamente insólito, desde la óptica de lo científico, y presupuestando que muy a pesar de este proceder el paciente falleciera, la investigación penal por el hecho dañoso, con fundamento en la culpa.

El derecho a una muerte digna. Un Estado social de derecho como lo es Colombia, entra a proteger en sus asociados el derecho a la vida, tal como lo prevé el artículo segundo, inciso segundo respecto de los fines esenciales del Estado:

"las autoridades de la república están instituidas para proteger a todas las personas residentes en Colombia, en su vida, honra, bienes, creencias y demás derechos y libertades, y para asegurar el cumplimiento de los deberes sociales del Estado y de los particulares" A su turno, la Corte Constitucional ${ }^{10}$ ha llegado un poco

9 Cachón Pinzón, A. J. (2003). Fundamentos de la responsabilidad médica. Medellín: Ediciones Jurídicas Gustavo Ibáñez.

10 Colombia, Corte Constitucional. (1993, noviembre). "Sentencia T-571”, MP Sanín Greiffenstein, J. Bogotá. 
más lejos en este sentido a la vez que ha referenciado el principio de la dignidad humana en los siguientes términos:

"la integridad física, psíquica y espiritual, la salud, el mínimo de condiciones materiales necesarias para la existencia digna, son elementos constitutivos de una vida integra y presupuesto necesario para la autorrealización individual y social".

¿Es posible aceptar, entonces, que si el Estado tiene el deber jurídico de proteger la vida de los ciudadanos, ellos puedan autónomamente en momentos de precariedad extrema de su estado de salud decidir con la asistencia de un médico su continuidad o no? El Código Penal Colombiano en el artículo 326 ha tipificado como delito el homicidio por piedad en los siguientes términos:

"El que matare por piedad, para ponerse sus intensos sufrimientos provenientes de la lesión corporal o enfermedad grave e incurable incurrirá en prisión de seis meses a tres años".

Con base en sendas demandas la Corte Constitucional recibió una acción de inconstitucionalidad sobre justamente este artículo, por la cual, el demandante aducía lo inconstitucional de ese artículo por ir contra los principios del derecho a la vida de la Constitución y como consecuencia se disminuía el valor de la vida de un enfermo incurable, al de una persona en perfecto estado de salud como lo hacía el homicidio simple. La Corte concluyó que no se estaba violando la constitución marcando un precedente (jurisprudencia) en este tema. Pero esto permitió que la Corte se diera cuenta de las consecuencias que podía tener el consentimiento de la víctima por los cuales resultaría una razón para despenalizar la eutanasia si participa este factor.

De lo planteado en esta parte del escrito, se deriva que la eutanasia consentida por el enfermo, se convertiría en un derecho más, que no acarrearía responsabilidad alguna al profesional de la salud que la realizare previo seguimiento de los protocolos médicos que para tal efecto deben diligenciarse. Queda claro pues, que así como se protege constitucional y legalmente el derecho a la vida, al paciente en estado terminal también le asiste el derecho a la muerte digna; tomar la decisión de no prolongar innecesariamente su agonía, es el basamento del principio y, a la vez, derecho a la autodeterminación, es reconocer al enfermo en la relación médico- paciente.

\section{REFERENCIAS}

Cachón Pinzón, A. J. (2003). Fundamentos de la responsabilidad médica. Medellín. Ediciones jurídicas Gustavo Ibáñez.

Charlesworth, M. (1996). La bioética en una sociedad liberal. Edit. Cambridge University Press. Trad. Mercedes González.

Colombia, Corte Constitucional. (1995). Sentencia T-477” M. P. Martínez Caballero, A. Bogotá. 
Colombia, Corte Constitucional.(1993, Noviembre). Sentencia T-571”, M. P. Sanin Greiffenstein, J. Bogotá.

Francesc, A. (1995). Comités de Bioética: necesidad, estructura y funcionamiento. Labor hospitalaria. 40, 229; 136

Franco Zuloaga, J. A., \& Agudelo Yepes, M. C. El consentimiento y la advertencia de riesgo en medicina, Revista Instituto nacional de medicina en Colombia Vol. XVI

Galán Cortés, J. C. Doctor en medicina, abogado universidad de Oviedo. España.

REVISTA CUBANA DE ENFERMERÍA, (2006). REYES LÓPEZ, Madelin et al. Consentimiento informado en pacientes sometidos a cirugía por mínimo acceso. Rev Cubana Enfermer [online]. 2006, vol.22, n.4, pp. 0-0. ISSN 0864-0319

Europa, Tribunal Supremo, (1997, Febrero). Francia. 